\title{
Pseudoscalar Goldstone Bosons Scattering off Charmed Baryons with Chiral Perturbation Theory
}

\author{
Zhan-Wei Liu ${ }^{\mathrm{a}, *}$, Shi-Lin Zhu ${ }^{\mathrm{a}}$ \\ ${ }^{a}$ Department of Physics and State Key Laboratory of Nuclear Physics and Technology, Peking University, Beijing 100871, China
}

\begin{abstract}
We have systematically calculated the pseudoscalar Goldstone boson and charmed baryon scattering lengths to the third order with heavy baryon chiral perturbation theory. The scattering lengths can reveal the interaction of $K \Lambda_{c}, K \Sigma_{c}, \pi \Omega_{c}$ etc. For each channel, we take into account of the interaction between the external particle and every possible charmed baryon in the triplet, sextet, and excited sextet. We use dimensional regularization and modified minimal subtraction to get rid of the divergences in the loop-diagram corrections. We notice that the convergence of chiral expansion for most of the channels becomes better after we let the low energy constants absorb the analytic contributions of the loop-diagram corrections and keep the nonanalytic terms only.
\end{abstract}

(C) 2012 Published by Elsevier Ltd.

Keywords: chiral perturbation theory, charmed baryon

\section{Introduction}

There has been a growing interest in the properties of charmed hadrons after Belle, BABAR, CDF and other collaborations observed a series of interesting new states [1, 2, 3]. Much attention is paid to the recently observed charmed hadrons [4, 5, 6, 7, 8, 9, 10]. One may also wonder whether there exist some undiscovered new states. Certainly, the interaction of charmed hadrons is important to understand the underlying structure of these states.

The light pseudoscalar meson and charmed baryon scattering can reflect the interaction between them. We will use the heavy baryon chiral perturbation theory $(\mathrm{HB} \chi \mathrm{PT})$ to study the scattering lengths between the light pseudoscalar meson and charmed baryon in the triplet, sextet, and excited sextet to the third order. The scattering lengths are expanded as a power series in $\epsilon$ with explicit power counting, where $\epsilon$ is the small momentum of the light pseudoscalar mesons or the residual momentum of the charmed baryons. The mass difference between charmed baryons is also counted as $O\left(\epsilon^{1}\right)$.

The scattering length $a_{\phi B}$ is related to the $T$-matrix $T_{\phi B}$ at the threshold by $T_{\phi B}=4 \pi\left(1+m_{\phi} / m_{B}\right) a_{\phi B}$. On the one hand, the positive scattering length indicates that there exists attractive interaction in these channels, which might lead to the loosely bound molecular states. On the other hand, one can extract the scattering length with the observed molecular states to test the theory or determine the unknown low-energy constants [11, 12].

This paper is organized as follows: in Sec. 2, we list the $\mathrm{HB} \chi \mathrm{PT}$ Lagrangians of the pseudoscalar mesons and charmed baryons. In Sec. 3, we show the expressions of the scattering lengths. In Sec. 4, we discuss the numerical results. 


\section{Lagrangians}

The leading scattering length is at $O\left(\epsilon^{1}\right)$, and receives the tree-level contributions from Weinberg-Tomozawa terms of the leading Lagrangian only. The scattering length at $O\left(\epsilon^{2}\right)$ receives the tree-level contributions from the Lagrangian at $O\left(\epsilon^{2}\right)$. In addition to the tree-level contributions by the Lagrangian at $O\left(\epsilon^{3}\right)$, the scattering length at $O\left(\epsilon^{3}\right)$ also receives the one-loop corrections from the leading Lagrangians.

The leading $\mathrm{HB} \chi \mathrm{PT}$ Lagrangians read

$$
\begin{aligned}
\mathcal{L}_{\phi \phi}^{(2)}= & f^{2} \operatorname{Tr}\left(u_{\mu} u^{\mu}+\frac{\chi_{+}}{4}\right), \\
\mathcal{L}_{B \phi}^{(1)}= & \frac{1}{2} \operatorname{Tr}\left[\bar{B}_{\overline{3}} i v \cdot D B_{\overline{3}}\right]+\operatorname{Tr}\left[\bar{B}_{6}\left(i v \cdot D-\delta_{2}\right) B_{6}\right]-\operatorname{Tr}\left[\bar{B}_{6}^{*}\left(i v \cdot D-\delta_{3}\right) B_{6}^{*}\right] \\
& +2 g_{1} \operatorname{Tr}\left(\bar{B}_{6} S \cdot u B_{6}\right)+2 g_{2} \operatorname{Tr}\left(\bar{B}_{6} S \cdot u B_{\overline{3}}+\text { H.c. }\right)+g_{3} \operatorname{Tr}\left(\bar{B}_{6 \mu}^{*} u^{\mu} B_{6}+\text { H.c. }\right) \\
& +g_{4} \operatorname{Tr}\left(\bar{B}_{6 \mu}^{*} u^{\mu} B_{\overline{3}}+\text { H.c. }\right)+2 g_{5} \operatorname{Tr}\left(\bar{B}_{6}^{*} S \cdot u B_{6}^{*}\right)+2 g_{6} \operatorname{Tr}\left(\bar{B}_{\overline{3}} S \cdot u B_{\overline{3}}\right),
\end{aligned}
$$

where $v_{\mu}=(1, \overrightarrow{0})$ is the velocity of a slowly moving charmed baryon, $S_{\mu}$ is the spin matrix, and $\delta_{i}$ is the mass difference between charmed baryons

$$
\delta_{1}=M_{B_{6}^{*}}-M_{B_{6}}=67 \mathrm{MeV}, \quad \delta_{2}=M_{B_{6}}-M_{B_{\overline{3}}}=127 \mathrm{MeV}, \quad \delta_{3}=M_{B_{6}^{*}}-M_{B_{\overline{3}}}=194 \mathrm{MeV} .
$$

$B_{\overline{3}}, B_{6}$, and $B_{6}^{*}$ represent the charmed triplet, sextet, and excited sextet, respectively. The notations about the light pseudoscalar $\phi$ mesons are

$$
\Gamma_{\mu}=\frac{i}{2}\left[\xi^{\dagger}, \partial_{\mu} \xi\right], \quad u_{\mu}=\frac{i}{2}\left\{\xi^{\dagger}, \partial_{\mu} \xi\right\}, \quad \xi=\exp \left(i \frac{\phi}{2 f}\right), \quad \chi_{ \pm}=\xi^{\dagger} \chi \xi^{\dagger} \pm \xi \chi \xi, \quad \chi=\operatorname{diag}\left(m_{\pi}^{2}, m_{\pi}^{2}, 2 m_{K}^{2}-m_{\pi}^{2}\right) .
$$

$i D^{\mu} B_{a b}=i \partial^{\mu} B_{a b}+\Gamma_{a}^{\mu d} B_{d b}+\Gamma_{b}^{\mu d} B_{a d}$, which generates the Weinberg-Tomozawa $B B \phi \phi$ vertices. The terms proportional to $g_{i}$ in $\mathcal{L}_{B \phi}^{(1)}$ generate the axial coupling $B B \phi$ vertices.

The Lagrangians at $O\left(\epsilon^{2}\right)$ and $O\left(\epsilon^{3}\right)$ contain the counter terms and recoil terms. The counter terms are constructed based on the chiral symmetry, Lorentz invariance, $\mathrm{C}$ and $\mathrm{P}$ invariance, and so on, while the recoil terms are derived by the leading relativistic Lagrangians. They generate the $B B \phi \phi$-like vertices. The concrete form of the Lagrangians can be found in Ref. [13].

\section{Scattering Lengths}

We can get the scattering length to $O\left(\epsilon^{3}\right)$ by calculating the corresponding tree diagrams and loop diagrams in Fig. 1. The diagrams in Fig. 1(a) are made up of the Weinberg-Tomozawa vertices, while the diagrams in Fig. 1(b) are made up of two axial coupling vertices. We use the dimensional regularization and modified minimal subtraction to deal with the divergence generated by loop diagrams. All the divergence will be canceled after the redefinition of the low energy constants at $O\left(\epsilon^{3}\right)$ and renormalization of the wavefunction [13].

With the strict isospin symmetry, there are eleven independent channels for $\phi B_{\overline{3}}$ scattering, and nineteen independent channels for either $\phi B_{6}$ or $\phi B_{6}^{*}$ scattering. We can express the scattering length order by order. For example, the scattering length $a_{\pi \Sigma_{c}}^{(2)}$ in the $\pi \Sigma_{c}$ channel with the isospin 2 is

$$
\begin{aligned}
a_{\pi \Sigma_{c}}^{(2)}= & \frac{1}{4 \pi\left(1+m_{\pi} / m_{\Sigma_{c}}\right)}\left\{-\frac{m_{\pi}}{f_{\pi}^{2}}\right\}+\frac{1}{4 \pi\left(1+m_{\pi} / m_{\Sigma_{c}}\right)}\left\{-\frac{m_{\pi}^{2}\left(24 c_{0}+4 c_{1}-3 c_{2}-6 c_{4}\right)}{6 f_{\pi}^{2}}-\frac{m_{\pi}^{2}\left(g_{1}^{2}+2 g_{2}^{2}\right)}{8 M_{0} f_{\pi}^{2}}\right\} \\
& +\frac{1}{4 \pi\left(1+m_{\pi} / m_{\Sigma_{c}}\right)}\left\{\left[-\frac{m_{\pi}^{3}\left(g_{1}^{2}+2 g_{2}^{2}\right)}{16 f_{\pi}^{2} M_{0}^{2}}+\frac{m_{\pi}^{2} \delta_{2}\left(g_{1}^{2}+g_{2}^{2}\right)}{8 f_{\pi}^{2} M_{0}^{2}}\right]\right. \\
& \left.+\left[-\frac{1}{8} V\left(m_{K}^{2},-m_{\pi}\right)-\frac{1}{2} V\left(m_{\pi}^{2},-m_{\pi}\right)-\frac{1}{4} V\left(m_{\pi}^{2}, m_{\pi}\right)-\frac{1}{9} m_{\pi}^{2} W_{2}\left(m_{\eta}\right)+m_{\pi}^{2} W_{2}\left(m_{\pi}\right)\right]\right\},
\end{aligned}
$$



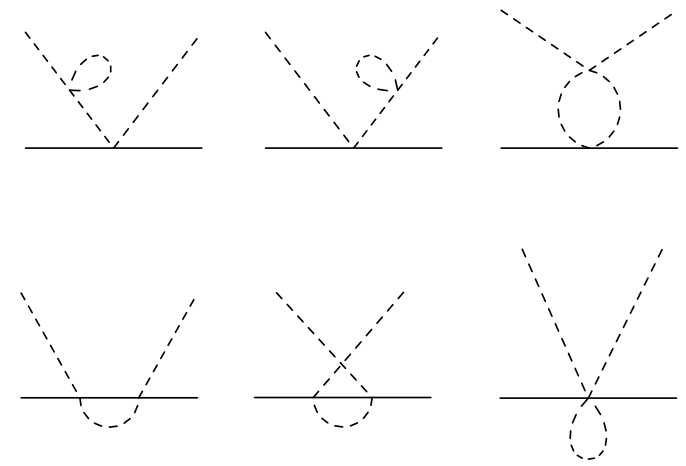

(a) no axial vertices
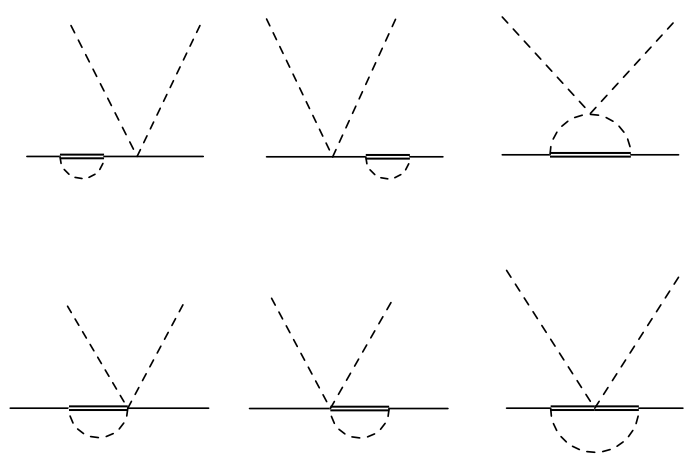

(b) two axial vertices

Figure 1. Nonvanishing loop diagrams for the pseudoscalar meson and charmed meson scattering lengths to $O\left(\epsilon^{3}\right)$ with HB $\chi \mathrm{PT}$. The dashed lines represent the pseudoscalar Goldstone bosons. Both the thin solid lines and thick solid lines represent charmed baryons. The internal thin solid lines represent the charmed baryons in the same representation as the external baryons while the internal thick solid lines represent all possible charmed baryons.

where $M_{0}$ is the mean mass of charmed triplet baryons, and $c_{i}$ is the LEC at $O\left(\epsilon^{2}\right)$. The scalar functions $V$ and $W_{2}$ come from the loop correction, and their definitions can be found in Ref. [13].

To get the final numerical results, we should determine the LECs of Lagrangians. One obtains $\left|g_{2}\right|=0.60$ and $\left|g_{4}\right|=1.0$ by fitting the decay widths of $\Sigma_{c}$ and $\Sigma_{c}^{*}[14]$. Due to lack of the experimental data, we cannot fit all the LECs. One can get $\left|g_{1}\right|=\sqrt{8 / 3}\left|g_{2}\right|$ with the quark model symmetry, and $\left|g_{3}\right|=\sqrt{3} / 2\left|g_{1}\right|,\left|g_{5}\right|=3 / 2\left|g_{1}\right|$, $\left|g_{6}\right|=0$ with heavy quark spin symmetry. We use the SU(4) flavor approximation to roughly determine the LECs at $O\left(\epsilon^{2}\right)$,

$$
c_{0}=-0.79 \mathrm{GeV}, \quad c_{1}=-0.98 \mathrm{GeV}, \quad c_{2}=-2.07 \mathrm{GeV}-2 \frac{\alpha^{\prime}}{4 \pi f}, \quad c_{3}=\frac{\alpha^{\prime}}{4 \pi f}, \quad c_{4}=-0.84 \mathrm{GeV},
$$

where we take the dimensionless constant $\alpha^{\prime}$ to be in the range of [-1,1] as in Ref. [15]. As for the LECs at $O\left(\epsilon^{3}\right)$, we use two different kinds of approximations. We first assume that the LECs at $O\left(\epsilon^{3}\right)$ are zero (Approximation I). We also let the LECs absorb the analytical corrections from loop diagrams totally (Approximation II). The analytical corrections are the polynomials of the momentum or mass of the charmed baryons and light pseudoscalar mesons.

\section{Results and discussions}

Now we can get the scattering lengths of 49 independent channels to the third order [13]. From our numerical results, we notice that there exists attractive interaction in the following channels with the positive real parts of scattering lengths: $\pi \Xi_{c}{ }^{(1 / 2)}, K \Xi_{c}^{(0)}, K \Xi_{c}{ }^{(1)}, \bar{K} \Lambda_{c}{ }^{(1 / 2)}, \bar{K} \Xi_{c}{ }^{(0)}, \eta \Lambda_{c}{ }^{(0)}, \eta \Xi_{c}{ }^{(1 / 2)}, \pi \Xi_{c}^{(1 / 2)}, \pi \Sigma_{c}{ }^{(0)}, \pi \Sigma_{c}{ }^{(1)}, K \Omega_{c}^{(1 / 2)}, K \Xi_{c}^{(0)}$, $K \Xi_{c}^{\prime(1)}, K \Sigma_{c}{ }^{(1 / 2)}, \bar{K} \Xi_{c}^{\prime(0)}, \bar{K} \Sigma_{c}^{(1 / 2)}, \bar{K} \Sigma_{c}{ }^{(3 / 2)}, \eta \Omega_{c}{ }^{(0)}, \eta \Xi_{c}^{\prime(1 / 2)}, \eta \Sigma_{c}{ }^{(1)}, \pi \Xi_{c}^{*(1 / 2)}, \pi \Sigma_{c}^{*(0)}, \pi \Sigma_{c}^{*(1)}, K \Omega_{c}^{*(1 / 2)}, K \Xi_{c}^{*(0)}, K \Xi_{c}^{*(1)}$, $K \Sigma_{c}^{*(1 / 2)}, \bar{K} \Xi_{c}^{*(0)}, \bar{K} \Sigma_{c}^{*(1 / 2)}, \bar{K} \Sigma_{c}^{*(3 / 2)}, \eta \Omega_{c}^{*(0)}, \eta \Xi_{c}^{*(1 / 2)}$, and $\eta \Sigma_{c}^{*(1)}$, where the superscripts in parentheses refer to the isospin.

There is no great difference of the scattering lengths between the two different approximations of the LECs at $O\left(\epsilon^{3}\right)$ in most of the channels. There are 41 channels, in each of which the difference between the different approximations is at most $20 \%$ of the smaller scattering length. The convergence of the chiral expansion with Approximation II is better than that with Approximation I generally, especially in the following channels $\bar{K} \Lambda_{c}{ }^{(1 / 2)}, K \Omega_{c}{ }^{(1 / 2)}, K \Sigma_{c}{ }^{(3 / 2)}, \bar{K} \Omega_{c}{ }^{(1 / 2)}$, $\bar{K} \Sigma_{c}^{(1 / 2)}, K \Omega_{c}^{*(1 / 2)}, K \Sigma_{c}^{*(3 / 2)}, \bar{K} \Omega_{c}^{*(1 / 2)}$, and $\bar{K} \Sigma_{c}^{*(1 / 2)}$.

We investigate the effect of the mass difference $\delta_{i}$ between charmed baryons. The mass difference $\delta_{i}$ only affects the scattering lengths at $O\left(\epsilon^{3}\right)$. In Table. 1, we list the $\phi B_{\overline{3}}$ scattering lengths at $O\left(\epsilon^{3}\right)$ with $\delta_{i}$ in Eq. (3) and with $\delta_{i}=0$. From the table, we notice that the difference with different $\delta_{i}$ is at most $10 \%$ of the smaller scattering length in seven channels among the total ten channels. The effect of the mass difference for the $\phi B_{6}$ and $\phi B_{6}^{*}$ scattering lengths is small in most of the channels similar to that for the $\phi B_{\overline{3}}$ scattering lengths. 


\begin{tabular}{c|cc|cc}
\hline & \multicolumn{2}{|c|}{$\delta_{1}=67, \delta_{2}=127, \delta_{3}=194(\mathrm{MeV})$} & \multicolumn{2}{c}{$\delta_{1}=\delta_{2}=\delta_{3}=0$} \\
& Approximation I & Approximation II & Approximation I & Approximation II \\
\hline$a_{\pi \Lambda_{c}}^{(1)}$ & -0.008 & -0.017 & -0.02 & -0.02 \\
$a_{\pi \Xi^{(1 / 2)}}$ & 0.011 & 0.00044 & 0.0079 & -0.0027 \\
$a_{\pi \Xi_{c}}^{(3) 2)}$ & -0.048 & -0.043 & -0.051 & -0.046 \\
\hline$a_{K \Lambda_{c}}^{(1 / 2)}$ & -0.24 & -0.15 & -0.24 & -0.15 \\
$a_{K \Xi_{c}}^{(0)}$ & 0.052 & -0.090 & 0.095 & -0.091 \\
$a_{K \Xi_{c}}^{(1)}$ & $0.27+0.18 i$ & $0.23+0.18 i$ & $0.25+0.18 i$ & $0.25+0.18 i$ \\
$a_{\bar{K} \Lambda_{c}}^{(1 / 2)}$ & $0.084+0.27 i$ & $-0.0071+0.27 i$ & $0.084+0.27 i$ & $-0.0071+0.27 i$ \\
$a_{\bar{K} \Xi_{c}}^{(0)}$ & 0.93 & 0.75 & 0.88 & 0.78 \\
$a_{\bar{K} \Xi_{c}}^{(1)}$ & -0.24 & -0.15 & -0.23 & -0.14 \\
\hline$a_{\eta \Lambda_{c}}^{(0)}$ & $0.095+0.19 i$ & $0.083+0.19 i$ & $0.088+0.19 i$ & $0.088+0.19 i$ \\
$a_{\eta \Xi_{c}}^{(1 / 2)}$ & $0.036+0.098 i$ & $0.028+0.098 i$ & $0.026+0.098 i$ & $0.026+0.098 i$ \\
\hline \hline
\end{tabular}

Table 1. the $\phi B_{\overline{3}}$ scattering lengths at $O\left(\epsilon^{3}\right)$ with different mass difference $\delta_{i}$ in units of fm

In summary, we have calculated the pseudoscalar meson and charmed baryon scattering lengths to $O\left(\epsilon^{3}\right)$ with $\mathrm{HB} \chi \mathrm{PT}$. There exists attractive interaction in some channels, which might lead to the formation of some loosely bound molecules. We notice that the convergence becomes better in most of the channels with the non-analytic approximation. The effect of the mass difference between charmed baryons is not large. Hopefully our results will be useful for the chiral extrapolation of lattice QCD simulation of the pion heavy baryon strong interaction.

\section{Acknowledgments}

This work is supported by the National Natural Science Foundation of China under Grants 11075004, 11021092 and Ministry of Science and Technology of China(2009CB825200).

\section{References}

[1] B. Aubert, et al. (BABAR Collaboration), Phys. Rev. Lett. 90 (2003) 242001

[2] P. Krokovny, et al. (Belle Collaboration), Phys. Rev. Lett. 91 (2003) 262002.

[3] T. Aaltonen, et al. (CDF Collaboration), Phys. Rev. Lett. 102 (2009) 242002.

[4] C.-Y. Wong, Phys. Rev. C 69 (2004) 055202.

[5] E. Kolomeitsev and M. Lutz, Phys. Lett. B 582 (2004) 39

[6] D. Gamermann, E. Oset, D. Strottman, and M. J. V. Vacas, Phys. Rev. D 76 (2007) 074016.

[7] Y.-B. Dai, X.-Q. Li, S.-L. Zhu, and Y.-B. Zuo, Eur. Phys. J. C 55 (2008) 249.

[8] X. Liu and S. Zhu, Phys. Rev. D 80 (2009) 017502.

[9] X. Liu, Z.-G. Luo, Y.-R. Liu, and S.-L. Zhu, Eur. Phys. J. C 61 (2009) 411.

[10] O. Romanets, et al., Phys. Rev. D 85 (2012) 114032.

[11] V. Baru, et al., Phys. Lett. B 586 (2004) 53.

[12] U. G. Meißner, (2012) arXiv: 1211.3026.

[13] Z.-W. Liu and S.-L. Zhu, Phys. Rev. D 86 (2012) 034009.

[14] W. Meguro, Y.-R. Liu, and M. Oka, Phys. Lett. B 704 (2011) 547

[15] F. Guo, C. Hanhart, and U. Meißner, Eur. Phys. J. A 40 (2009) 171. 